\title{
Effects of Brucine on the OPG/RANKL/RANK Signaling Pathway in MDA-MB-231 and MC3T3-E1 Cell Coculture System
}

\author{
Ruixian Wu, ${ }^{1}$ Qian $\mathrm{Li}^{2}{ }^{2}$ Xiaohua Pei, ${ }^{2}$ and Kefei $\mathrm{Hu}^{3}$ \\ ${ }^{1}$ Third Clinical Medical College, Beijing University of Chinese Medicine, Beijing 100029, China \\ ${ }^{2}$ The Third Affiliated Hospital, Beijing University of Chinese Medicine, Beijing 100029, China \\ ${ }^{3}$ Beijing Yuan Lai Health Technology Development Co. Ltd., Beijing 100035, China \\ Correspondence should be addressed to Xiaohua Pei; pxh_127@163.com
}

Received 6 June 2017; Revised 11 August 2017; Accepted 20 August 2017; Published 26 September 2017

Academic Editor: Kamal D. Moudgil

Copyright (C) 2017 Ruixian Wu et al. This is an open access article distributed under the Creative Commons Attribution License, which permits unrestricted use, distribution, and reproduction in any medium, provided the original work is properly cited.

\begin{abstract}
The present study examined the effects of brucine on the OPG/RANKL/RANK signaling pathway for exploring the mechanism of brucine suppression of bone metastasis in breast cancer. MDA-MB-231 breast cancer cells and mouse osteoblast MC3T3-E1 cells were cocultured to mimic the breast cancer bone metastasis microenvironment in vitro. qRT-PCR and Western blotting were used to detect the expressions of OPG and RANKL at the mRNA and protein levels, respectively, in brucine-treated cultures and they were compared to those in untreated cultures. We aimed to understand the effect of brucine on the entire OPG/RANKL/RANK signaling pathway after analyzing these effects. Results showed that brucine treatment significantly increased both the OPG mRNA/RANKL mRNA expression ratio and the OPG protein/RANKL protein ratio in cocultures compared to those in untreated cocultures $(P<0.01)$. Brucine, therefore, plays a regulatory role in the OPG/RANKL/RANK signaling pathway, suggesting that it can indirectly control osteoclasts by regulating the expression and secretion of OPG and RANKL in osteoblast cells, thereby inhibiting the differentiation and bone resorption function of osteoclasts.
\end{abstract}

\section{Introduction}

Breast cancer is the leading cause of cancer-related mortality in women, with half million deaths annually worldwide [1]. More than $90 \%$ of these deaths are caused by metastasis [2], as approximately $65-75 \%$ of the patients with metastatic breast cancer develop bone metastases [3, 4]. Breast cancer bone metastasis is closely associated with osteoclastogenesis and osteolytic bone metastasis [5]; however, the precise molecular mechanisms are not fully understood.

Semen Strychni was first documented in the Compendium of Materia Medica and was believed to be capable of removing tissue masses owing to its ability to promote blood circulation and granulation and remove necrotic tissue. For these reasons, it has been used in cancer treatment for a long time. Brucine is the major active ingredient in Semen Strychni. Extensive research has been conducted in the past few years to investigate the antineoplastic effect of brucine $[6,7]$. Several researchers have shown that Semen Strychni has a therapeutic effect in hepatoma cell lines (e.g., SMMC7221, HepG2, and H22), breast cancer cell lines (e.g., MDAMB-231 and MCF-7), and hematological tumor cell lines (e.g., K562 and U266), among others. Some researchers have also suggested that brucine might inhibit the growth of bone metastases in breast cancer of nude mice and alleviate bone destruction $[8,9]$. Therefore, the effect of brucine on bone metastases in breast cancer has attracted attention.

The observation that the OPG/RANKL/RANK system plays a vital role in osteoclastogenesis has been a significant breakthrough in the field of bone physiology [10-12]. The OPG/RANKL/RANK system plays a critical role in maintaining bone balance, which determines whether osteolytic metastasis would be initiated. In this study, we examined the key factors in this system, namely, OPG and RANKL, in an in vitro coculture model using the MDA-MB-231 breast cancer and the mouse osteoblast MC3T3-E1 cell lines. Specifically, we examined the interaction between the breast cancer cells and osteoblasts in a microenvironment that mimicked bone 
TABle 1: Primer sequences.

\begin{tabular}{lc}
\hline Name & Primer sequence \\
\hline OPG upstream primer & $5^{\prime}$-ATGGACAACCCAGGAAACCC-3' \\
OPG downstream primer & $5^{\prime}$-GTAGGTGCCAGGAGCACATT-3 \\
RANKL upstream primer & $5^{\prime}$-ATGATGGAAGGCTCATGGTTGG-3' \\
RANKL downstream primer & $5^{\prime}$-CAGCATTGATGGTGAGGTGTG-3' \\
GAPDH upstream primer & $5^{\prime}$-AGCCTTCCTTCTTGGGTATG-3' \\
GAPDH downstream primer & $5^{\prime}$-GGTCTTTACGGATGTCAACG-3' \\
\hline
\end{tabular}

metastases in breast cancer and assessed the effect of brucine, using alterations in the mRNA and protein levels of OPG and RANKL as readouts.

\section{Materials and Methods}

2.1. Cells. The human breast cancer cell line MDA-MB-231 and mouse osteoblast cell line MC3T3-E1 (Shanghai Cell Bank of the Chinese Academy of Sciences, Shanghai, China) were used.

2.2. Reagents. Fetal bovine serum (HyClone, Logan, Utah, USA), trypsin (Gibco, Grand Island, NY, USA), $\alpha$-modified Eagle's medium (MEM) (Life Technologies Corporation, NY, USA, lot number 1406320), 2x Taq PCR Master Mix (dye included), Taq polymerase, SYBR Green PCR Master Mix (Invitrogen Corporation, Carlsbad, CA, USA), DEPC (diethyl pyrocarbonate), DEPC-treated water (DNase/RNase-free double distilled $\mathrm{H}_{2} \mathrm{O}$ ) (Amresco, Solon, OH, USA), anti-rabbit IgG HRP-linked (7074S, Cell Signaling Technology, Danvers, MA, USA), rabbit anti-goat IgG-HRP (sc-2768, Santa Cruz, USA), and Western blot marker (color predyed) (Thermo Fisher Scientific) were used.

2.3. Drugs. Brucine (molecular formula: $\mathrm{C}_{23} \mathrm{H}_{26} \mathrm{~N}_{2} \mathrm{O}_{4}$ ) was purchased from the National Institute for Food and Drug Control (lot number 110706-201306; purity: 91.7\%). Zoledronic acid injection was manufactured by Novartis Pharma Schweiz AG (catalog number S0051; specification: $100 \mathrm{~mL}$; $5 \mathrm{mg})$.

2.4. Instruments. Eco Real-Time PCR system (Illumina, San Diego, CA, USA), high-speed centrifuge, pipettes (range: $2.5 \mu \mathrm{L}$ to $1000 \mu \mathrm{L}$ ) (Eppendorf, Hamburg, Germany), VE-180 vertical electrophoresis unit, VE-186 Trans-Blot transfer unit (Tanon Science and Technology, Shanghai, China), vortex shaker (model: HYQ-3110; Crystal Technology \& Industries, Dallas, Texas, USA), microplate reader (model: ELX800; BioTek Laboratories, Vermont, USA), and ultrasonic cleaner (model: SB-5200DTDN; Scientz, Ningbo, China) were used.

2.5. Experimental Groups. For the model group, the human breast cancer cell line MDA-MB-231 and the mouse osteoblast MC3T3-E1 cell line were cocultured in the presence of an induction medium ( $\alpha$-MEM) containing $\beta$-glycerophosphate $(10 \mathrm{mmol} / \mathrm{L})$ and L-ascorbic acid
$(50 \mu \mathrm{g} / \mathrm{mL})$. The control group was the same as the model group minus the MDA-MB-231 breast cancer cells. The brucine group with $0.02 \mathrm{mmol} / \mathrm{L}$ is a model group treated with $0.02 \mathrm{mmol} / \mathrm{L}$ brucine. The brucine group with $0.04 \mathrm{mmol} / \mathrm{L}$ is a model group treated with $0.04 \mathrm{mmol} / \mathrm{L}$ brucine. The brucine group with $0.08 \mathrm{mmol} / \mathrm{L}$ is a model group plus $0.08 \mathrm{mmol} / \mathrm{L}$ brucine. The positive control drug group is a model group plus $10 \mu \mathrm{mol} / \mathrm{L}$ zoledronic acid.

2.6. Coculture of MDA-MB-231 and MC3T3-E1 Cells. The MC3T3-E1 mouse osteoblast cell line was cultured in $\alpha$ MEM as a single cell suspension. The cell concentration was adjusted to $1 \times 10^{6} / \mathrm{mL}$ and the suspension was inoculated into a T-75 culture flask and cultured until the MC3T3E1 cells reached approximately $80 \%-90 \%$ confluence. The medium was then replaced with $\alpha$-MEM induction medium containing $\beta$-glycerophosphate $(10 \mathrm{mmol} / \mathrm{L})$ and L-ascorbic acid $(50 \mu \mathrm{g} / \mathrm{mL})$. Six days after induction, the breast cancer cell MDA-MB-231, which had been grown as a single cell suspension and adjusted to a cell concentration of $5.6 \times$ $10^{4} / \mathrm{mL}$, was added directly to the culture flask. The cells were then cocultured for seven days with different experimental media as described above. The media were replaced after every two days.

2.7. Quantitative Real-Time Polymerase Chain Reaction ( $q R T$ $P C R$ ). Total RNA was extracted using the TRIzol kit (Life Technologies, NY, USA) according to the manufacturer's instructions. A small amount of RNA was removed to determine its optical density (OD) value and the remainder was stored at $-80^{\circ} \mathrm{C}$.

The $20 \mu \mathrm{L}$ PCR reaction system included $1 \mu \mathrm{L}$ primer, $11 \mu \mathrm{L}$ nuclease-free ultrapure water, $4 \mu \mathrm{L} 5 \mathrm{x}$ reaction buffer, $1 \mu \mathrm{L}$ RiboLock $^{\mathrm{TM}}$ RNA enzyme inhibitor $(20 \mathrm{U} / \mu \mathrm{L})$, $2 \mu \mathrm{L} 10 \mathrm{mM}$ dNTP mix, and $1 \mu \mathrm{L}$ RevertAid $^{\mathrm{TM}} \mathrm{M}-\mathrm{MuLV}$ reverse transcriptase $(200 \mathrm{U} / \mu \mathrm{L})$ (Thermo Fisher Scientific, Waltham, MA, USA).

Fluorescent quantitative PCR was conducted as follows: in a PCR tube, the cDNA template $(1 \mu \mathrm{L})$, upstream primer $(1 \mu \mathrm{L}, 10 \mu \mathrm{M})$, downstream primer $(1 \mu \mathrm{L}, 10 \mu \mathrm{M})$, SYBR green $(10 \mu \mathrm{L})$, and double-distilled $\mathrm{H}_{2} \mathrm{O}(7 \mu \mathrm{L})$ were mixed together to obtain a total reaction volume of $20 \mu \mathrm{L}$. PCR was performed using the following cycle: $50^{\circ} \mathrm{C}$ for $2 \mathrm{~min}, 95^{\circ} \mathrm{C}$ initial denaturation for $10 \mathrm{~min}, 95^{\circ} \mathrm{C}$ denaturation for $15 \mathrm{~s}$, and $60^{\circ} \mathrm{C}$ annealing for $30 \mathrm{~s}$, for a total of 40 cycles. The primer sequences used are shown in Table 1. 
2.8. Western Blotting. Drugs were added to cocultures of MDA-MB-231 and MC3T3-E1 cells for $24 \mathrm{~h}$ as described above. Total protein was extracted from the cells using radioimmunoprecipitation assay (RIPA) lysis buffer (Solarbio, Beijing, China). Equal amounts of protein extracts were then separated using $10 \%$ sodium dodecyl phosphatepolyacrylamide gel electrophoresis (SDS-PAGE) (Gibco, Grand Island, USA) and transferred onto a polyvinylidene fluoride (PVDF) membrane $(0.45 \mu \mathrm{m})$ (Millipore, Bedford, MA, USA). The membranes were blocked with $5 \% \mathrm{w} / \mathrm{v}$ nonfat dry milk (Gibco, Grand Island, USA) dissolved in Trisbuffered saline (Amresco, Solon, OH, USA) plus Tween20 (TBS-T) (Gibco, Grand Island, USA) at $25^{\circ} \mathrm{C}$ for $1 \mathrm{~h}$, followed by overnight incubation with primary antibodies at $4^{\circ} \mathrm{C}$. The primary antibodies for immunoblotting were antiOPG antibody (P-17) (sc-21038, Santa Cruz, CA, USA), antiRANKL antibody (ab124797, Abcam, Cambridge, UK), and anti- $\beta$-actin antibody (N-21) (sc-130657, Santa Cruz, USA). After washing with TBS-T (CoWin Biotech, Beijing, China), the membranes were incubated with HRP-labeled secondary antibodies (Cell Signaling Technologies, Danvers, MA, USA) for $1 \mathrm{~h}$ at $25^{\circ} \mathrm{C}$. The membranes were analyzed using a protein visualizer ECL (Tanon Science and Technology, Shanghai, China).

2.9. Statistical Analysis. All experiments were repeated at least thrice. SPSS 20.0 data analysis software was used for statistical analysis and processing. Kruskal-Wallis $H$-test was employed for the nonparametric test of two or more independent samples. All data are expressed as the mean \pm standard deviation. $P$ values less than $0.05(P<0.05)$ were considered statistically significant.

\section{Results}

3.1. Effect of Brucine on OPG and RANKL $m$ RNA Levels. qRTPCR was used to measure OPG and RANKL mRNA levels in cocultures of the human breast cancer cell line MDA-MB-231 and the mouse osteoblast MC3T3-E1 cell line with or without brucine. The model group's OPG and RANKL mRNA levels were significantly higher than those of the control group $(P<$ $0.01)$. Brucine $(0.04$ and $0.08 \mathrm{mmol} / \mathrm{L})$ increased $O P G$ and RANKL mRNA levels significantly compared to the model group $(P<0.01$ or $P<0.05)$, as shown in Figures 1 and 2 .

Figures 1 and 2 show comparison of OPG and RANKL mRNA levels in different experimental groups. qRT-PCR was used to measure the OPG and RANKL mRNA levels. MDAMB-231 and MC3T3-E1 cells were cocultured for 7 days; different doses of brucine $(0.02,0.04$, and $0.08 \mathrm{mmol} / \mathrm{L})$ and $10 \mu \mathrm{mol} / \mathrm{L}$ zoledronic acid were given (Figure $1,{ }^{\# \#} P<0.01$, compared to the control group; ${ }^{* *} P<0.01$, compared to the model group; Figure 2, ${ }^{\#} P<0.01$, compared to the control group; ${ }^{* *} P<0.05$, compared to the model group).

\subsection{Effect of Brucine on OPG $m R N A / R A N K L m R N A$ Ratio.} Since osteoblasts secrete both OPG and RANKL, which are required for bone protection and bone destruction, respectively, the ratio of OPG/RANKL more accurately reflects the

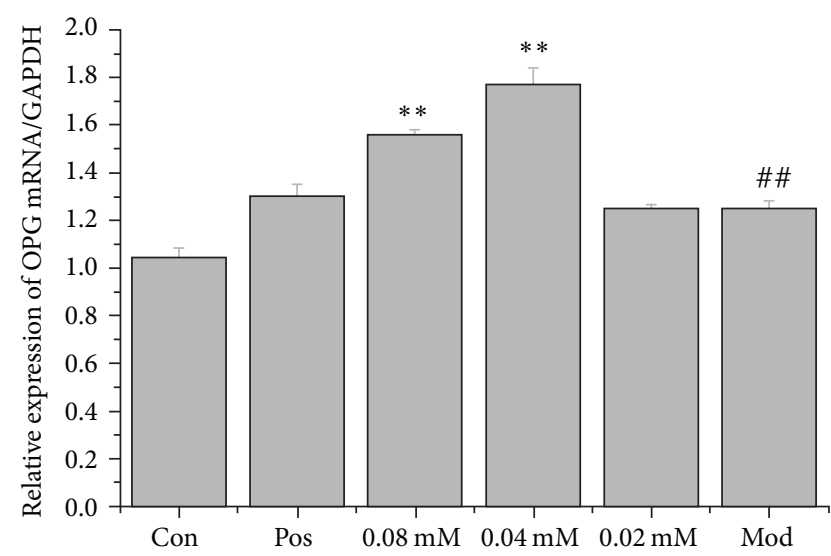

FIGURE 1: Comparison of OPG mRNA levels in different experimental groups.

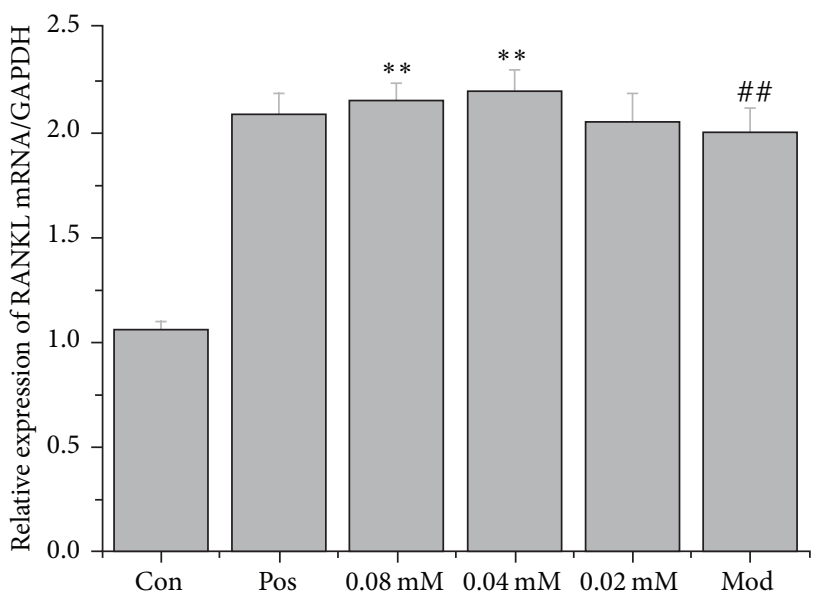

FIGURE 2: Comparison of RANKL mRNA levels in different experimental groups.

balance between bone absorption and bone reconstruction. Therefore, we analyzed and compared the ratio of OPG mRNA/RANKL mRNA in the different experimental groups. Results showed that the OPG mRNA/RANKL mRNA ratio of the model group was significantly lower compared to that of the control group $(P<0.01)$. Brucine $(0.04$ and $0.08 \mathrm{mmol} / \mathrm{L})$ increased the OPG mRNA/RANKL mRNA ratio significantly compared to that in the model group $(P<$ 0.01) (Figure 3).

3.3. Effects of Brucine on OPG and RANKL Protein Levels. Western blot was used to measure the OPG and RANKL protein levels in different experimental groups. Results showed that OPG level was significantly lower in the model group compared to that in the control group $(P<0.01)$. In contrast, the model group's RANKL level was significantly higher than that of the control group $(P<0.01)$. Brucine $(0.02,0.04$, and $0.08 \mathrm{mmol} / \mathrm{L}$ ) and the positive control drug zoledronic acid significantly increased OPG levels and decreased RANKL levels, compared to those in the model group $(P<0.01)$ (Figures 4 and 5). 


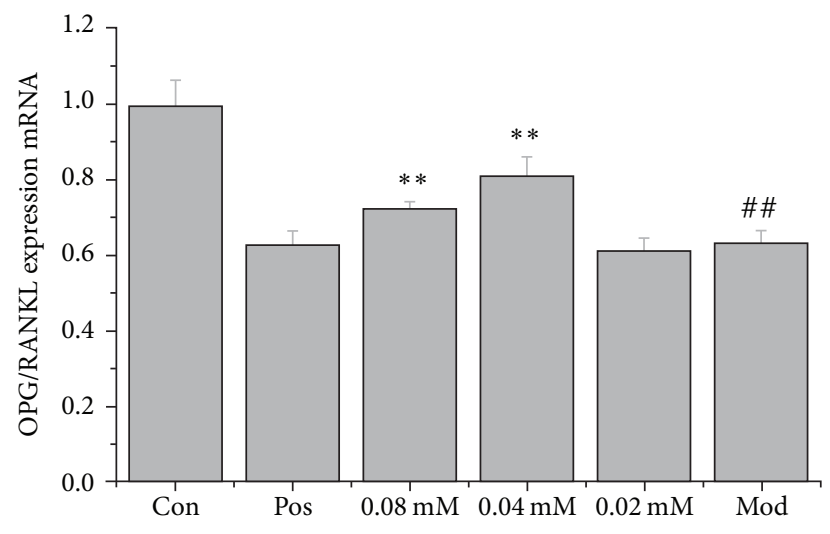

FIGURE 3: Comparison of the OPG mRNA/RANKL mRNA ratio in different experimental groups. Notes. ${ }^{\# \#} P<0.01$, compared to the control group; ${ }^{* *} P<0.01$, compared to the model group.

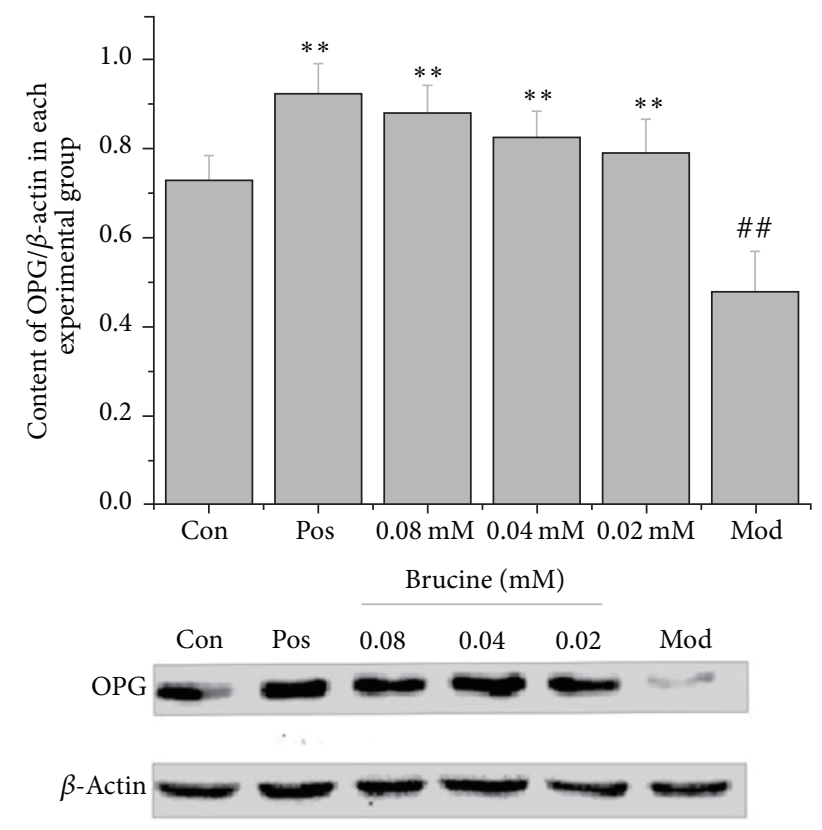

FIGURE 4: Comparison of OPG protein levels in different experimental groups.

Figures 4 and 5 show comparison of OPG and RANKL protein levels in different experimental groups. The Western blot method was used to measure the OPG and RANKL protein expression levels. MDA-MB-231 and MC3T3-E1 cells were cocultured for 7 days; different doses of brucine $(0.02$, 0.04 , and $0.08 \mathrm{mmol} / \mathrm{L}$ ) and $10 \mu \mathrm{mol} / \mathrm{L}$ zoledronic acid were given (Figure $4,{ }^{\# \#} P<0.01$, compared to the control group; ${ }^{* *} P<0.01$, compared to the model group; Figure $5,{ }^{\#} P<$ 0.01 , compared to the control group; ${ }^{* *} P<0.01$, compared to the model group).

3.4. Effects on the OPG/RANKL Protein Ratio. Similar to the analysis conducted for mRNA levels, we analyzed and compared the OPG protein/RANKL protein ratio in different experimental groups. Results showed that the OPG

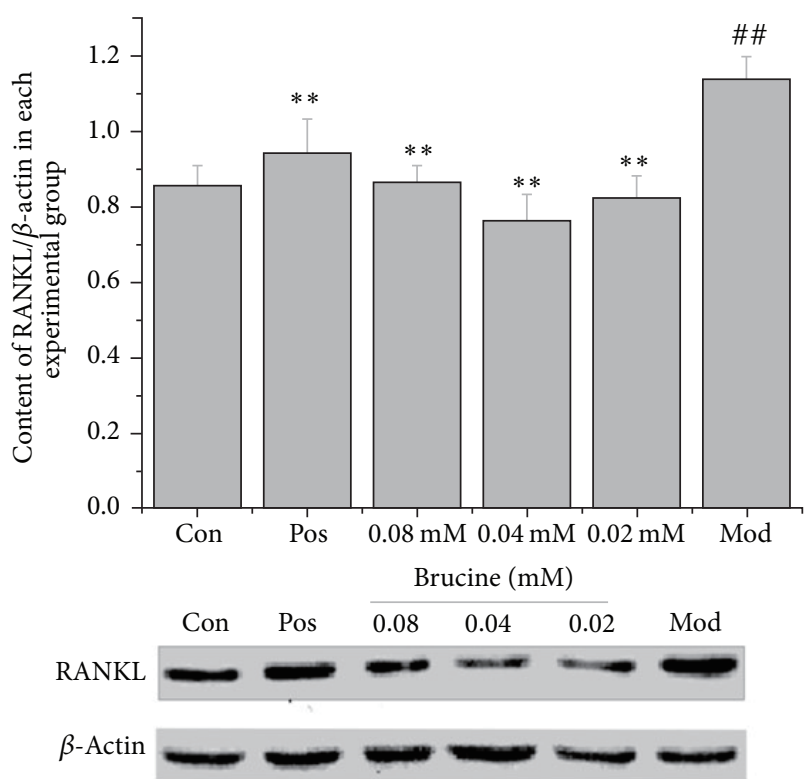

FIGURE 5: Histogram showing RANKL protein level in different experimental groups.

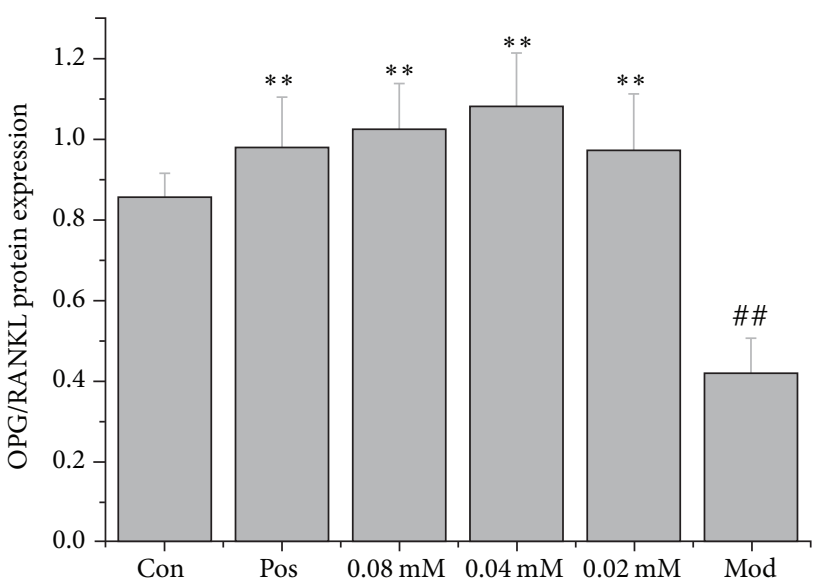

FIGURE 6: Comparison of the OPG protein /RANKL protein ratio in different experimental groups. Notes. ${ }^{\# \#} P<0.01$, compared to the control group; ${ }^{* *} P<0.01$, compared to the model group.

protein/RANKL protein ratio of the model group was significantly lower compared to that of the control group $(P<0.01)$. Brucine $(0.02,0.04$, and $0.08 \mathrm{mmol} / \mathrm{L})$ and the positive control drug zoledronic acid significantly increased the OPG/RANKL protein ratio compared to that in the model group $(P<0.01)$ (Figure 6).

\section{Discussion}

Breast cancer, along with lung and prostate cancers, is likely to metastasize to the bone [3]. Bone metastases worsen the prognosis, as chances of survival decrease and the quality of life of the patient dramatically deteriorates, with a clinical outcome characterized by intractable pain, nerve compression 
syndromes, increased risk of fractures, and hypercalcemia [13]. Certain drugs, including estrogen, bisphosphonates, teriparatide, denosumab, and synthetic calcitonin, have been used for the treatment of osteolysis. Most of these drugs have serious limitations or side effects, such as osteonecrosis, osteosarcoma, thromboembolism, and esophageal irritation [14-16]. Brucine is a bitter alkaloid extracted from the Strychnos nux-vomica tree, found in Southeast Asia. Several studies have shown that brucine is an effective agent for the treatment of breast cancer [17]. Intriguingly, brucine has been found to inhibit bone metastasis in breast cancer [18], vascular endothelial growth factor (VEGF) expression, and angiogenesis [8,19]; however, its precise mechanism of action remains unknown.

The process of bone metastasis in breast cancer is complex and arises due to the interaction between multiple cells in the bone microenvironment which communicate through the release of numerous cytokines. In this process, the OPG/RANKL/RANK signaling pathway is considered to be critical for adjusting bone metabolic balance. As a member of the tumor necrosis factor (TNF) superfamily of ligands, RANKL, which is expressed primarily by osteoblasts and bone marrow stromal cells and occurs membrane-bound on the surface of these cells, plays an important role in the activation of lymphocytes, dendritic cells, and osteoclasts $[20,21]$. RANK, the receptor for RANKL, is found on the cell membrane of lymphocytes, activated $\mathrm{T}$ cells, B cells, osteoclasts, and other cell types and is important for osteolysis and growth of the lymph node [22,23]. OPG is a member of the tumor necrosis factor receptor superfamily, which inhibits the differentiation of osteoclasts, and a reduction in its expression has been considered to be a key factor in the occurrence and development of bone metastases in breast cancer. Although OPG is widely expressed in a variety of tissues, it is functional only in the bone. Studies showed that osteoblasts regulate the differentiation of osteoclasts via the secretion and expression of OPG and RANKL [24]. Thus, the levels of OPG, RANKL, and RANK in the local microenvironment of bone tissues are considered as defining biological factors, which mediate a variety of molecules to induce the production and function of osteoclasts, and are also key determinants of the balance between bone destruction and formation [25-27].

Our previous study demonstrated that brucine inhibited osteoclastogenesis by suppressing the Jagged1/Notch1 signaling pathways [28]. In the current study, we demonstrate that brucine $(0.04$ and $0.08 \mathrm{mmol} / \mathrm{L})$ significantly increases both OPG and RANKL mRNA levels compared to those in the model group. Brucine also increases the OPG mRNA/RANKL mRNA ratio in cocultures of the human breast cancer cell line MDA-MB-231 and the mouse osteoblast MC3T3-E1 cell line. Different doses of brucine and the positive control drug zoledronic acid significantly increased OPG protein levels and decreased RANKL protein levels. However, brucine and zoledronic acid also significantly increased the OPG protein/RANKL protein ratio. These results suggest that brucine may regulate the bone metabolic balance by regulating OPG/RANKL/RANK signaling pathways.
Osteoclasts are giant multinuclear cells derived from the monocyte-macrophage lineage. Osteoclast differentiation from precursor cells can be induced by RANKL, which also controls the survival and function of mature osteoclasts. Therefore, RANKL has been used in studies on differentiation and function of osteoclasts. Comparison of the experimental data from the model and control groups showed that the introduction of the breast cancer cells decreased the OPG/RANKL ratio, which might have stimulated osteoclast activation and differentiation via increase in RANK-RANKL numbers and intracellular signal transduction, thereby disrupting bone metabolic balance. Comparison of the brucine data from the model groups showed that the introduction of the brucine increased the OPG/RANKL ratio. We conclude that brucine inhibited the OPG/RANKL/RANK signaling pathway by regulating both mRNA and protein levels. On one hand, brucine directly affected osteoclast differentiation, while, on the other hand, it inhibited osteoclast differentiation and maturation via indirect regulation of osteoclast function by osteoblasts.

\section{Conclusions}

In conclusion, brucine appears to play a regulatory role in the OPG/RANKL/RANK signaling pathway, suggesting that it can indirectly control osteoclasts possibly by regulating the OPG and RANKL levels secreted by osteoblast cells, thereby inhibiting the differentiation and bone resorption functions of osteoclasts. Further studies are required for verifying whether brucine can inhibit bone metastases of breast cancer in vivo and whether OPG and RANKL levels are related to bone metastases in brucine-treated breast cancer patients.

\section{Conflicts of Interest}

All authors declare that there are no conflicts of interest regarding the publication of this article.

\section{Authors' Contributions}

Xiaohua Pei contributed to the design of the study and analytic strategy; Ruixian $\mathrm{Wu}$ and Kefei $\mathrm{Hu}$ completed the experiment; Ruixian Wu and Qian Li analyzed the data and wrote the paper.

\section{Acknowledgments}

This work was supported by Beijing Natural Science Foundation (no. 7164273) and Beijing University of Chinese Medicine Project Foundation (no. 2015-JYB-JSMS142).

\section{References}

[1] J. Ferlay, E. M. SoerjomataramI, R. Dikshit, S. Eser, C. Mathers et al., "GLOBOCAN 2012 vl.0, cancer incidence and mortalityworldwide: IARC CancerBase No. 11," in International Agency for Research on Cancer, Lyon, France, 2013. 
[2] X. Jin and P. Mu, “Targeting Breast Cancer Metastasis," Breast Cancer: Basic and Clinical Research, vol. 9, pp. 23-34, 2015.

[3] R. E. Coleman, "Clinical features of metastatic bone disease and risk of skeletal morbidity, Clinical Cancer Research, vol. 12, no. 20, pp. 6243-6249, 2006.

[4] R. D. Rubens and I. Fogelman, Bone Metastases, Springer, London, UK, 1991.

[5] P. Clézardin, “Therapeutic targets for bone metastases in breast cancer," Breast Cancer Research, vol. 13, no. 2, article 207, 2011.

[6] X. K. Deng, B. C. Cai, W. Yin et al., "Study on antineoplastic effect and toxicity of Heps tumor-bearing mice," Chinese Pharmacological Bulletin, vol. 22, no. 1, pp. 35-38, 2006.

[7] Y. MA, Z. LI, Y. WANG, and J. FENG, "Brucine induces the apoptosis of U266 multiple myeloma cells by phosphorylation of c-Jun," Molecular Medicine Reports, vol. 7, no. 2, pp. 481-484, 2013.

[8] P. Li, M. Zhang, W. Ma, X. Sun, and F. Jin, "Effects of brucine on vascular endothelial growth factor expression and microvessel density in a nude mouse model of bone metastasis due to breast cancer," Chinese Journal of Integrative Medicine, vol. 18, no. 8, pp. 605-609, 2012.

[9] K. C. Xia, M. Zhang, and P. Li, "Inhibition mechanism of brucine by transdermal administration on bone metastases of breast cancer," Chinese Journal of Cancer Prevention and Treatment, vol. 19, no. 23, pp. 1771-1775, 2012.

[10] N. Giuliani, S. Colla, and V. Rizzoli, "New insight in the mechanism of osteoclast activation and formation in multiple myeloma: Focus on the receptor activator of NF- $\kappa \mathrm{B}$ ligand (RANKL)," Experimental Hematology, vol. 32, no. 8, pp. 685691, 2004.

[11] N. Giuliani, S. Colla, F. Morandi, S. Barille-Nion, and V. Rizzoli, "Lack of receptor activator of nuclear factor- $\kappa \mathrm{B}$ ligand (RANKL) expression and functional production by human multiple myeloma cells," Haematologica, vol. 90, no. 2, pp. 275278, 2005.

[12] U. Heider, I. Zavrski, C. Jakob et al., "Expression of receptor activator of NF-?B ligand (RANKL) mRNA in human multiple myeloma cells," Journal of Cancer Research and Clinical Oncology, vol. 130, no. 8, 2004.

[13] J. Engel, R. Eckel, J. Kerr et al., "The process of metastasisation for breast cancer," European Journal of Cancer, vol. 39, no. 12, pp. 1794-1806, 2003.

[14] A. Mariotti, "Bisphosphonates and osteonecrosis of the jaws.," Journal of dental education, vol. 72, no. 8, pp. 919-929, 2008.

[15] T. D. Rachner, S. Khosla, and L. C. Hofbauer, "Osteoporosis: now and the future," The Lancet, vol. 377, no. 9773, pp. 12761287, 2011.

[16] G. A. Rodan and T. J. Martin, “Therapeutic approaches to bone diseases," Science, vol. 289, no. 5484, pp. 1508-1514, 2000.

[17] L. M. Zhao, Y. G. Liu, and Z. X. Niu, "Anti-tumor effect on brucine," Chinese Journal of Cancer Prevention \& Treatment, vol. 20, pp. 877-880, 2013.

[18] W. J. Ma and P. Li, "Effects of brucine on breast cancer bone metastasis in mice," Anhui Med Pharm, vol. 13, pp. 600-602, 2009.

[19] F. J. Lowery and D. Yu, "Growth factor signaling in metastasis: current understanding and future opportunities," Cancer and Metastasis Reviews, vol. 31, no. 3-4, pp. 479-491, 2012.

[20] Y.-Y. Kong, H. Yoshida, and I. Sarosi, "OPGL is a key regulator of osteoclastogenesis, lymphocyte development and lymph-node organogenesis," Nature, vol. 397, no. 6717, pp. 315-323, 1999.
[21] N. Kim, P. R. Odgren, D. Kim, S. C. Marks, and Y. Choi, "Diverse roles of the tumor necrosis factor family member TRANCE in skeletal physiology revealed by TRANCE deficiency and partial rescue by a lymphocyte-expressed TRANCE transgene," Proceedings of the National Academy of Sciences, vol. 97, no. 20, pp. 10905-10910, 2000.

[22] W. C. Dougall, M. Glaccum, K. Charrier et al., "RANK is essential for osteoclast and lymph node development," Genes \& Development, vol. 13, no. 18, pp. 2412-2424, 1999.

[23] J. Li, I. Sarosi, and X.-Q. Yan, "RANK is the intrinsic hematopoietic cell surface receptor that controls osteoclastogenesis and regulation of bone mass and calcium metabolism," Proceedings of the National Academy of Sciences of the United States of America, vol. 97, no. 4, pp. 1566-1571, 2000.

[24] H. Goto, A. Hozumi, M. Osaki et al., "Primary human bone marrow adipocytes support TNF- $\alpha$-induced osteoclast differentiation and function through RANKL expression," Biomedical Research, vol. 56, no. 3, pp. 662-668, 2011.

[25] N. Udagawa, N. Sato, S. Yang et al., "Signal transduction of lipopolysaccharide-induced osteoclast differentiation," Periodontology 2000, vol. 43, no. 1, pp. 56-64, 2007.

[26] J.-M. Kim, D. Jeong, H. K. Kang, S. Y. Jung, S. S. Kang, and B.-M. Min, "Osteoclast precursors display dynamic metabolic shifts toward accelerated glucose metabolism at an early stage of RANKL-stimulated osteoclast differentiation," Cellular Physiology and Biochemistry, vol. 20, no. 6, pp. 935-946, 2007.

[27] A. Yamada, M. Takami, T. Kawawa et al., "Interleukin-4 inhibition of osteoclast differentiation is stronger than that of interleukin-13 and they are equivalent for induction of osteoprotegerin production from osteoblasts," Immunology, vol. 120, no. 4, pp. 573-579, 2007.

[28] K.-F. Hu, X.-Y. Kong, M.-C. Zhong, H.-Y. Wan, N. Lin, and X.H. Pei, "Brucine inhibits bone metastasis of breast cancer cells by suppressing Jagged1/Notchl signaling pathways," Chinese Journal of Integrative Medicine, vol. 23, no. 2, pp. 110-116, 2017. 


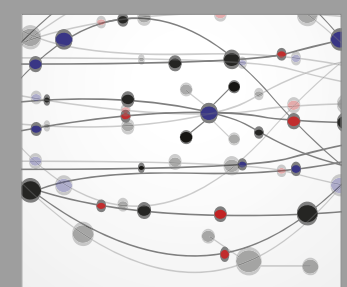

The Scientific World Journal
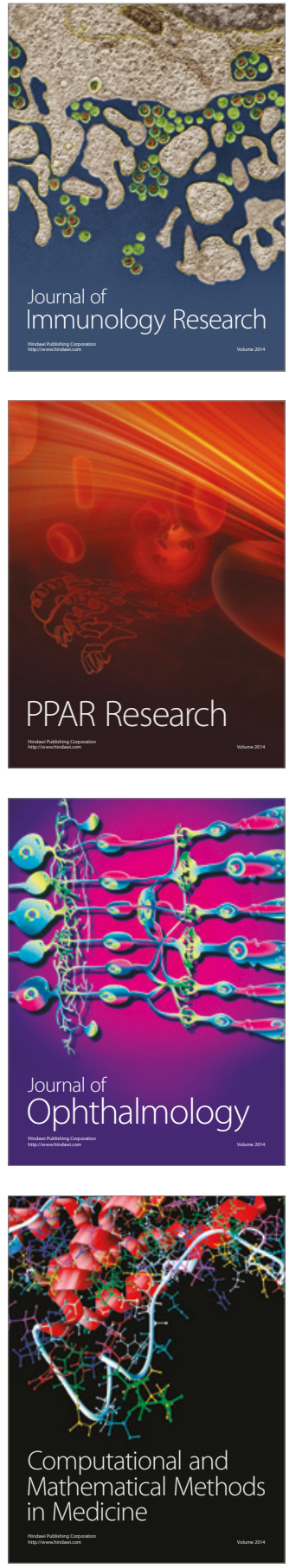

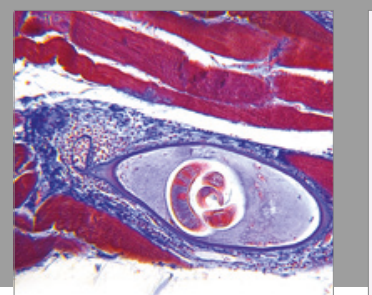

Gastroenterology Research and Practice
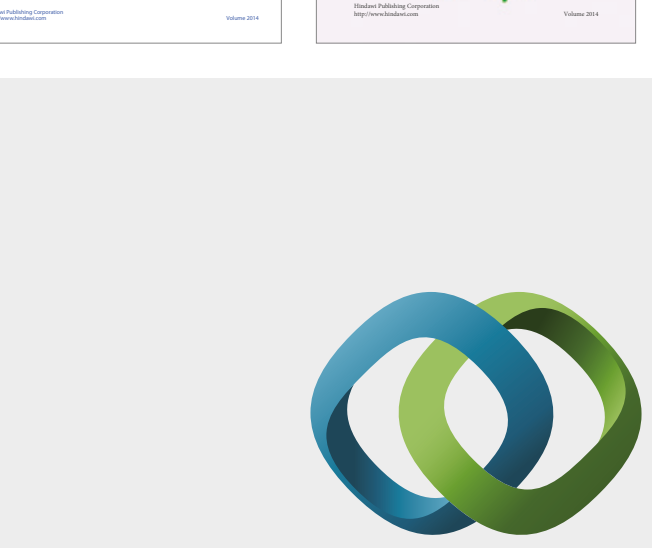

\section{Hindawi}

Submit your manuscripts at

https://www.hindawi.com
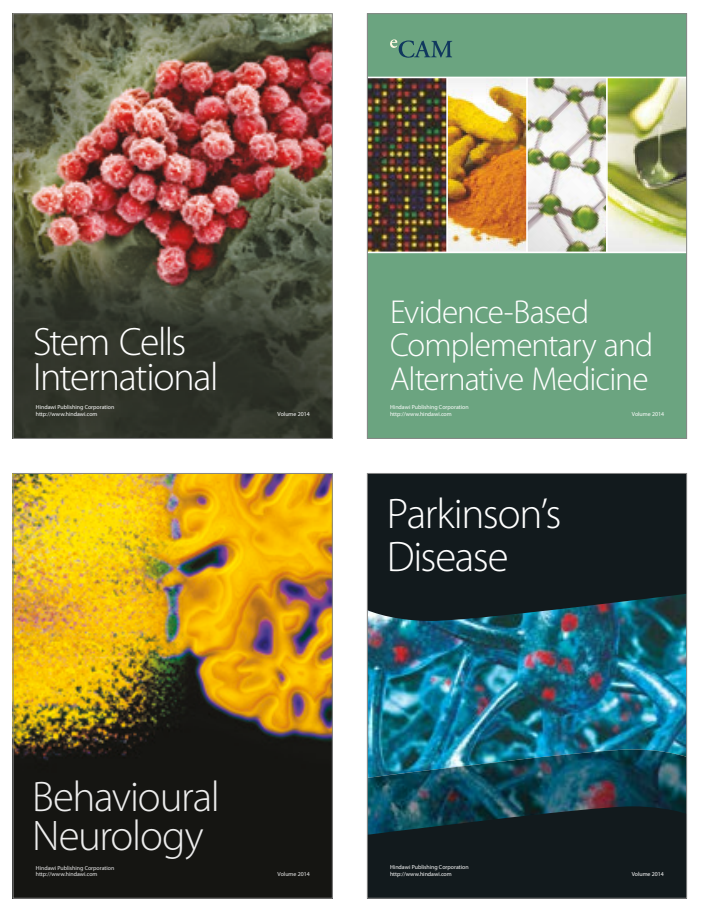
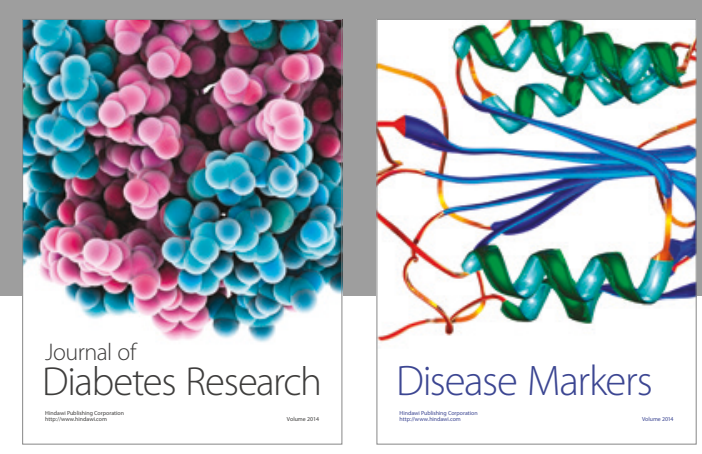

Disease Markers
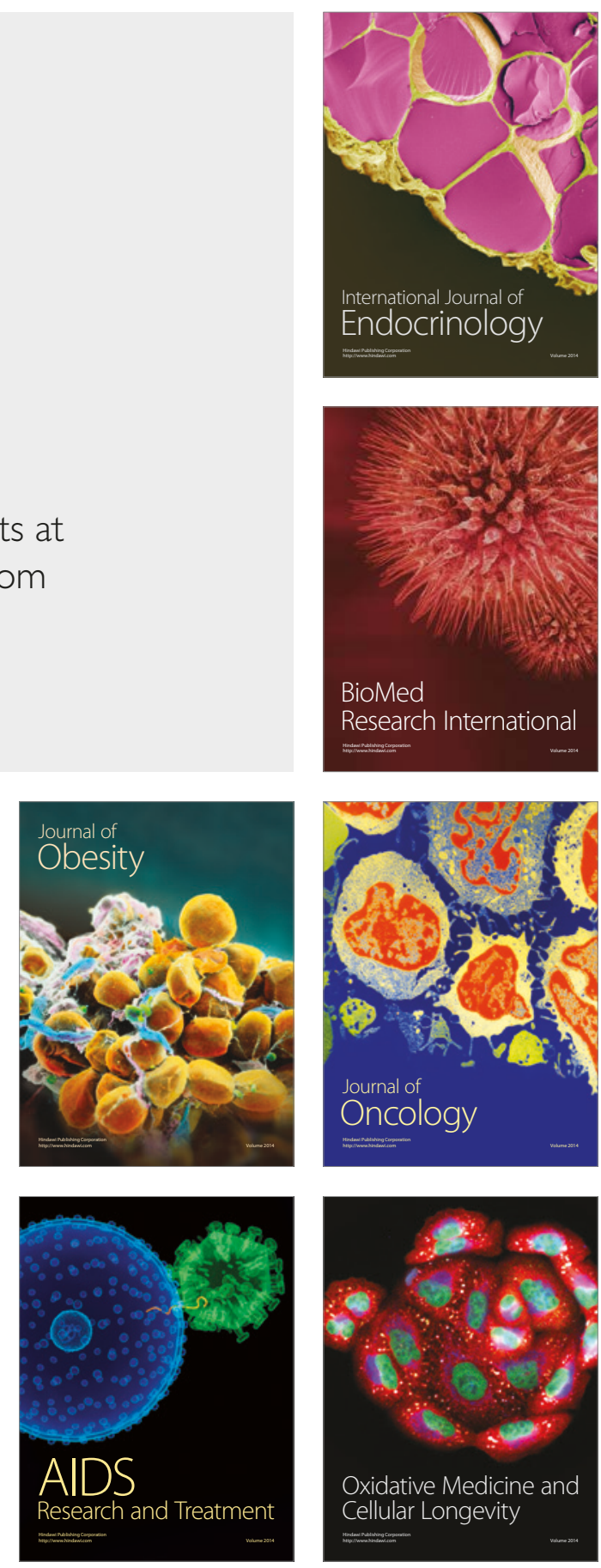